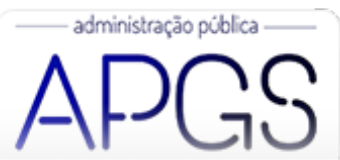

Administração Pública e Gestão Social ISSN: 2175-5787

apgs@ufv.br

Universidade Federal de Viçosa

Brasil

\title{
Motivação para coprodução do bem público: proposta de uma escala
}

Viana Bezerra, Joacil Carlos; Cavalcante, Carlos Eduardo; Gouveia Júnior, Antonio Motivação para coprodução do bem público: proposta de uma escala

Administração Pública e Gestão Social, vol. 14, núm. 1, 2022

Universidade Federal de Viçosa, Brasil

Disponible en: https://www.redalyc. org/articulo. $0 a ?$ id=351569604003

\section{(c) $(1) \Theta$}

Esta obra está bajo una Licencia Creative Commons Atribución-NoComercial-SinDerivar 4.0 Internacional. 


\title{
Motivação para coprodução do bem público: proposta de uma escala
}

\author{
Joacil Carlos Viana Bezerra \\ Universidade Federal da Paraíba, Brasil \\ joacilcarlosviana@gmail.com \\ Carlos Eduardo Cavalcante \\ Universidade Federal da Paraíba, Brasil \\ cavalcanteeduardo@gmail.com \\ Antonio Gouveia Júnior \\ Universidade Federal da Paraíba, Brasil \\ agouveiajunior@gmail.com
}

Motivation for the co-production of public goods: a scale proposal

Motivación para la coproducción del bien público: una propuesta a escala

Redalyc: https://www.redalyc.org/articulo.oa? id $=351569604003$

Recepción: 18 Diciembre 2020

Aprobación: 10 Agosto 2021

Publicación: 08 Enero 2022

\section{Resumo:}

Objetivo da pesquisa: O presente estudo teve como objetivo a construção de uma escala para mensurar os aspectos que motivam o cidadão a participar na coprodução do bem público (CBP), por meio das organizações da sociedade civil (OSC).

Enquadramento teórico: A pesquisa utiliza como base teórica diversas lentes da coprodução, porém, com enfoque na prestação de serviços públicos. Utiliza, ainda, a literatura sobre construção e validação de escalas.

Metodologia: $\mathrm{O}$ estudo percorreu etapas exploratórias e descritivas. A fase exploratória envolveu uma revisão integrativa da literatura e geração de itens. A fase descritiva detalhou os procedimentos utilizados para construção e validação da escala. Os dados foram analisados por meio software $\operatorname{SPSS}_{25^{\circ}}$ e R (pacote lavaan).

Resultados: A escala é composta por quatro dimensões e 12 itens, sendo gerada a partir de 174 questionários. Os resultados apontam, entre outras coisas: o cidadão acredita que sua participação na CBP melhora os serviços ofertados à sociedade; ele está mais disposto a coproduzir em atividades que beneficiam à comunidade onde reside; e participam porque veem outras pessoas participando.

Originalidade: $\mathrm{O}$ instrumento desenvolvido é capaz de facilitar a implementação do processo de coprodução, por refletir aspectos motivacionais do cidadão e apontar que não há um modelo ou formato único para os diferentes contextos da coprodução. O estudo avança na literatura da $\mathrm{CBP}$ ao tentar responder o seguinte questionamento: O que motiva o cidadão a participar na CBP por meio das OSC? O esforço realizado na pesquisa ajudará gestores públicos e sociais, bem como estudiosos do tema, a construírem repostas para a indagação.

Contribuições teóricas e práticas: Além de buscar preencher uma lacuna da literatura, a escala pode ser útil a gestores públicos e de OSC que pretendem implementar uma gestão baseada na CBP e pode informar sobre estratégias de incentivos utilizadas para aumentar a participação dos cidadãos na prestação de serviços públicos.

Palavras-CHave: Coprodução do bem público, participação cidadã, organizações da sociedade civil, escala.

\section{Abstract:}

Research objective: The present study aimed to build a scale to measure the aspects that motivate the citizen to participate in the co-production of the public good (CBP), through civil society organizations (CSOs).

Theoretical framework: The research uses several coproduction lenses as a theoretical basis, but with a focus on the provision of public services. It also uses the literature on scale construction and validation.

Methodology: The study went through exploratory and descriptive stages. The exploratory phase involved an integrative literature review and item generation. The descriptive phase detailed the procedures used to build and validate the scale. The data were analyzed using SPSS $25^{\circ}$ and R software (lavaan package). 
Results: The scale consists of 4 dimensions and 12 items, being generated from 174 questionnaires. The results show, among other things: the citizen believes that his participation in CBP improves the services offered to society; he is more willing to co-produce in activities that benefit the community where he resides; and participate because they see other people participating.

Originality: The instrument developed is capable of facilitating the implementation of the co-production process, as it reflects motivational aspects of the citizen and points out that there is no single model or format for the different forms of co-production. The study advances in the CBP literature when trying to answer the following question: What motivates the citizen to participate in CBP through CSOs? The effort made in the research will help both public and social managers, as well as scholars of the theme, to construct answers to the question.

Theoretical and practical contributions: In addition to seeking to fill a gap in the literature, the scale can be useful to public and CSO managers who intend to implement CBP-based management and can inform about incentive strategies used to increase citizen participation in the provision of services public.

KEYWORDS: Public services co-production, citizen participation, civil society organizations, scale.

\section{ReSUMEN:}

Objetivo de la investigación: El presente estudio tuvo como objetivo construir una escala para medir los aspectos que motivan al ciudadano a participar en la coproducción del bien público (PBC), a través de organizaciones de la sociedad civil (OSC).

Marco teórico: La investigación utiliza varios lentes de coproducción como base teórica, pero con un enfoque en la prestación de servicios públicos. También utiliza la literatura sobre construcción y validación de escalas.

Metodología: El estudio pasó por etapas exploratorias y descriptivas. La fase exploratoria implicó una revisión integradora de la literatura y la generación de ítems. La fase descriptiva detalló los procedimientos utilizados para construir y validar la escala. Los datos se analizaron mediante el software SPSS $25^{\circ}$ y R (paquete lavaan).

Resultados: La escala consta de 4 dimensiones y 12 ítems, siendo generada a partir de 174 cuestionarios. Los resultados muestran, entre otras cosas: el ciudadano cree que su participación en la CBP mejora los servicios que se ofrecen a la sociedad; está más dispuesto a coproducir en actividades que beneficien a la comunidad donde reside; y participar porque ven a otras personas participando.

Originalidad: El instrumento desarrollado es capaz de facilitar la implementación del proceso de coproducción, ya que refleja aspectos motivacionales del ciudadano y señala que no existe un modelo o formato único para las diferentes formas de coproducción. El estudio avanza en la literatura de la CBP al intentar dar respuesta a la siguiente pregunta: ¿Qué motiva al ciudadano a participar en la CBP a través de las OSC? El esfuerzo realizado en la investigación ayudará tanto a los gestores públicos y sociales, como a los estudiosos del tema, a construir respuestas a la pregunta.

Contribuciones teóricas y prácticas: Además de buscar llenar un vacío en la literatura, la escala puede ser útil para los administradores públicos y de las OSC que pretenden implementar la gestión basada en la PBC y puedan informar sobre las estrategias de incentivos utilizadas para aumentar la participación ciudadana en la prestación servicios. servicios públicos.

Palabras Clave: Coproducción de servicios públicos, participación ciudadana, organizaciones de la sociedad civil, escala.

\section{INTRODUÇÃO}

O cidadão pode participar do ciclo de políticas públicas e desempenhar um papel ativo na produção de bens e serviços públicos relevantes para ele e para a comunidade em geral. Esse papel ativo na produção de bens e serviços públicos foi caracterizado, por Ostrom (1996), como sendo coprodução. Na coprodução, a formulação das políticas deixa de ser vista como um processo impositivo e passa a ser encarada como uma integração entre o Estado e o cidadão. Tal integração faz com que o cidadão tenha mais informações sobre o processo e lhe "dá voz".

Algumas pesquisas sobre coprodução apontam quem coproduz, o que coproduz e quais os efeitos da coprodução (Verschuere, Brandsen, \& Pestoff, 2012; Bovaird, Ryzin, Loeffler, \& Parrado, 2015). Seguindo essa mesma linha, alguns estudos realizados no Brasil apontam os efeitos da coprodução (Silva, Knol, \& Moretto, 2016; Age \& Schommer, 2017; Rocha, Schommer, Debetir, \& Pinheiro, 2019), quem coproduz (Ronconi, Debetir, \& Mattia, 2011; Schommer, Nunes, \& Moraes, 2012; Ribeiro, Andion, \& Burigo, 2015; Soares \& Farias, 2019) ou ambos (Salm, Menegasso, \& Ribeiro, 2007; Klein, Salm, Heidemann, \& Menegasso, 2012; Chaebo \& Medeiros, 2017). 
No entanto, as pesquisas realizadas não abordam o que motiva o cidadão para a CBP, apesar de o tema ser apontado como sugestão de pesquisas (Alford, 2002; Verschuere et al., 2012; Van Eijk \& Steen, 2014; Cepiku \& Giordano, 2014; Fledderus, Brandsen \& Honingh, 2015). Isso denota que há um campo de pesquisa em processo de sedimentação sobre o aspecto da motivação para a CBP que merece maior atenção, dado o fato que, de sobremaneira, impacta na questão do engajamento e respectivamente no desenho e desempenho do processo coprodutivo.

Como um esforço inicial na busca de suprir essa lacuna e visando a ampliar o conhecimento sobre a motivação para coprodução, foi realizada uma revisão integrativa da literatura, utilizando-se as seguintes bases de dados: Web of Science, Scopus, Scientific Electronic Library Online - SciELO e o Portal de periódicos da Capes. O acesso às bases foi realizado no período entre janeiro e junho de 2019. As buscas foram realizadas por "título" e por "resumo", sem o filtro "idioma” e "período de publicação", resultando em 162 artigos selecionados e 21 artigos analisados na revisão.

Não se observou a presença de trabalhos no contexto brasileiro que abordassem a motivação para coprodução, tampouco encontrou-se estudo com um instrumento capaz de mensurar os aspectos motivacionais para a coprodução. No entanto, acredita-se que para otimizar os processos de coprodução, os gestores públicos e das OSC precisam identificar quem são os coprodutores, que expectativas eles têm e o que lhes motiva a se engajar.

Assim, esta pesquisa se propõe a contribuir empírica e teoricamente com o campo da motivação para a $\mathrm{CBP}$, construindo uma escala para mensuração dos aspectos que motivam o cidadão a participar na CBP, por meio das OSC. O instrumento poderá auxiliar o Estado e as OSC no desenvolvimento de ações visando aumentar a participação cidadã.

\section{COPRODUÇÃO DO BEM PÚBLICO}

Durante as décadas de 1970 e 1980, nos Estados Unidos, estudiosos argumentavam que os cidadãos, enquanto apenas clientes, receberiam serviços mais efetivos e eficientes se fossem prestados somente por funcionários profissionais (Brandsen \& Pestoff, 2006). Ostrom (1996) apontou que a produção de um serviço era difícil sem a participação ativa daqueles que, supostamente, seriam beneficiados. Assim, começaram os esforços para o desenvolvimento do conceito de coprodução.

O conceito inicial foi desenvolvido por Elinor Ostrom e membros da Universidade de Indiana (EUA), no final da década de 1970. Eles definiram o termo coprodução como sendo uma relação que poderia existir entre o "produtor regular" - como os policiais, os professores ou os trabalhadores da saúde - e seus clientes, que queriam ser beneficiados pelo serviço e se sentirem mais seguros, mais instruídos ou mais saudáveis. Assim, definiram coprodução como um mix de atividades que tanto os profissionais do setor público como os cidadãos, combinam esforços na realização de serviços públicos (Ostrom, 1996).

Os primeiros conceitos eram centrados apenas no indivíduo e no agente do serviço público, o que representava uma limitação conceitual. Alford (2002), por sua vez, considerou a ampliação de agentes coprodutores e trouxe um conceito que incluía usuários, voluntários, organizações voluntárias ou comunitárias e outras organizações governamentais. Esses 'agentes' estão presentes no conceito de Schommer et al. (2012) ao apontar a CBP como uma estratégia de produção de bens e serviços públicos em redes e parcerias, contando com engajamento mútuo de governos e cidadãos, individualmente ou em torno de organizações associativas ou econômicas.

Diante dos conceitos apresentados, percebe-se que a produção dos serviços não é mais uma atividade exclusiva do Estado, dos seus servidores ou agentes públicos. Ela pode ser coproduzida por usuários, cidadãos e organizações. Dada a variedade e diversidade das organizações, algumas podem ser mais adequadas para promover a coprodução do que outras (Brandsen \& Pestoff, 2006; Pestoff, 2012). 
A promoção da coprodução está relacionada diretamente com a participação. Quanto maior for o grau de participação do cidadão, maior será a capacidade da organização em promover a coprodução (Ostrom, 1996; Pestoff, 2012) que se apresenta como um instrumento relevante de mudanças, uma vez que pode, entre outros exemplos, aumentar o envolvimento entre cidadãos e governos para a provisão de bens e serviços, trazer melhorias na prestação dos serviços públicos, promover efetividade nas políticas públicas e atingir metas sociais em várias áreas (Alford, 2002; Pestoff, 2012; Bovaird et al., 2015).

Além de analisar os efeitos citados anteriormente, alguns estudos sugerem que pesquisas futuras tenham como objetivo a identificação do que motiva o cidadão a coproduzir (Alford, 2002; Verschuere et al., 2012; Van Eijk \& Steen, 2014; Fledderus et al., 2015).

\section{MOTIVAÇÕES PARA COPRODUÇÃO DO BEM PÚBLICO.}

Para Alford (2002), existem diferentes motivações e diferentes níveis de disposição do indivíduo em coproduzir, fazendo com que a coprodução aconteça em diferentes contextos, sob os mais variados aspectos motivacionais. A partir de literaturas sobre motivação de clientes e voluntários, o autor buscou conceituar os fatores que podem influenciar na coprodução e cita cinco possíveis motivadores da coprodução: (I) sanções, (II) recompensas materiais, (III) recompensas intrínsecas, (IV) incentivos solidários e (V) recursos normativos.

Pesquisa realizada por Pestoff (2012) trouxe aspectos não materiais como sendo facilitadores do envolvimento do cidadão na coprodução. Esses aspectos são relacionados, entre outras coisas, com a distância até o provedor de serviços e as informações sobre o serviço a ser desenvolvido. Ainda sobre aspectos não materiais Petukiene, Tijunaitiene e Damkuviene (2012) realizaram um estudo na Lituânia e trazem que os relacionamentos construídos sobre confiança mútua, o respeito, a crença no poder da comunidade e a união são aspectos que podem motivar o cidadão a coproduzir junto com o Estado. Esses aspectos estão relacionados com os 'incentivos solidários' apontados por Alford (2002), demonstrando que, por vezes, os estudos analisam o mesmo aspecto, porém, com terminologias diferentes.

Ainda sobre terminologia, temos recompensas intrínsecas (Alford, 2002) e motivações intrínsecas (Vanleene, Verschuere, \& Voets, 2015) que partem da ideia de que alguém participa de certas atividades porque acha as atividades interessantes, dignas e agradáveis. Assim, motivações intrínsecas podem ser entendidas como forte gatilho para a CBP. No entanto, para termos uma visão mais ampla sobre as motivações dos cidadãos em coproduzir, é necessário pesquisar as coproduções em diferentes contextos e organizações (Van Eijk \& Steen, 2014).

Setecentos e noventa e sete participantes de diferentes organizações de bairro em Atlanta (Estados Unidos), fizeram parte da pesquisa de Uzochukwu e Thomas (2018) que propôs um conjunto de hipóteses sobre o envolvimento do cidadão na $\mathrm{CBP}$ abordando aspectos como eficácia, capacidade, motivação de serviço público, desejo de fazer a diferença, senso de dever cívico, contexto da coprodução e necessidades pessoais. $\mathrm{O}$ estudo aponta o senso de eficácia pessoal (se sentir capaz de fazer a diferença) como o maior aspecto motivacional entre os participantes. A motivação de serviço público (sentir que era seu dever) foi o segundo aspecto indicado e se sentir conectado com a comunidade, o terceiro.

Reforçando a temática, Verschuere et al. (2012) apontam que além da motivação individual das pessoas para coproduzir, outras circunstâncias influenciam a coprodução, como por exemplo: a sociabilidade, a satisfação com o serviço prestado, os objetivos do cidadão, a importância do serviço, as condições de realização do serviço e as iniciativas de envolvimento desenvolvidas pelo Estado ou pelas Organizações da Sociedade Civil. 


\section{METODOLOGIA}

Baseado nas sugestões de pesquisas e visando alcançar os objetivos propostos, o estudo percorreu etapas exploratórias e descritivas. A fase exploratória envolveu uma revisão integrativa da literatura e a geração de itens para a escala. A fase descritiva detalhou os procedimentos utilizados para construção da escala e foi dividida em: pré-teste da escala, caracterização dos procedimentos de coleta de dados, apresentação e análise dos resultados, validade e confiabilidade do construto.

A construção e validação da escala sobre motivação da CBP, seguiu os 10 passos propostos por Costa (2011) e utilizou como base os modelos teóricos desenvolvidos por Van Eijk e Steen (2014), Alford e Yates (2016) e Vanleene et al. (2017). Os 10 passos para a construção da escala estão descritos na seção de construção da escala e discussão dos resultados.

A pesquisa usou uma escala de verificação do tipo Likert de 10 pontos, com variação de 1 (discordância total) a 10 (concordância total). O questionário a ser respondido, na primeira e segunda amostragem, foi enviado por e-mail e aplicativo de mensagens WhatsApp para algumas OSC situadas fora da região metropolitana de João Pessoa e Campina Grande. Nessas duas regiões, os questionários foram entregues presencialmente para que pudéssemos frisar a importância do estudo, realizado por pesquisadores da Universidade Federal da Paraíba, junto aos integrantes das OSC. A coleta de dados da primeira amostragem, voluntários em geral, foi realizada no período entre 01 e 20 de outubro de 2019 , obtendo-se 370 respostas. $\mathrm{O}$ segundo instrumento de coleta de dados foi aplicado entre 12 de novembro e 14 de dezembro de 2019 com cidadãos que coproduzem serviços públicos por meio de OSC e foram obtidas 174 respostas.

\section{CONSTRUÇÃO DA ESCALA E DISCUSSÃO DOS RESULTADOS}

A construção da escala e a análise dos resultados obtidos ao longo deste estudo, compreenderam o detalhamento dos passos propostos por Costa (2011), mediante realização de análises exploratórias preliminares, análise de correlação, análise fatorial exploratória (AFE), análise fatorial confirmatória (AFC), análise de confiabilidade e validade de construto das dimensóes presentes na escala. Os procedimentos adotados são detalhados a seguir.

\section{Passo 1 - Especificação do domínio do construto}

Após a revisão integrativa da literatura, definiu-se que a motivação para a CBP seria o construto a ser analisado. Foram definidas as dimensões a serem analisadas e a decisão pela natureza refletiva dos itens, aqueles que têm sua variação afetada pela variação do construto.

Passo 2 - Atividades de geração de itens e validação de face e conteúdo

A revisão da literatura gerou 32 itens distribuídos em cinco dimensões:

a) Autoeficácia - Crença que o cidadão possui sobre sua capacidade em realizar um serviço ou exercer controle sobre determinados acontecimentos;

b) Natureza do serviço - Percepção que o cidadão possui sobre as características do serviço ou da organização na qual será prestado;

c) Sociabilidade - Recompensa e prazer que o cidadão tem de se associar com os outros, gerando conexões, redes sociais, reciprocidade e confiabilidade para a realização de determinados serviços;

d) Recompensas - Ações ou comportamentos que aumentam a sensação de satisfação no cidadão, fazendo com que ele possa desfrutar da experiência de participar da prestação de serviços;

e) Relevância percebida - Importância que o serviço tem para o cidadão que o presta e para quem o recebe.

Os 32 itens foram submetidos a validação de face e conteúdo por doutores em administração, estatística e gestão pública. A escolha dos especialistas se baseou na experiência em participação cidadã, gestão social e no desenvolvimento de escalas. Os especialistas realizaram a validade de face - quanto à clareza, pertinência 
e representatividade dos itens - e a validação de conteúdo, que é a verificação da adequação dos itens ao construto.

A avaliação de cada item foi realizada mediante instrumento de validação com uma gradação em cinco pontos. A adequação do item variou de 1 (inadequado) a 5 (adequação perfeita) e a clareza do enunciado com 1 (muito ruim) e 5 (muito bom). Após a análise dos especialistas, restaram os 28 itens constantes da Tabela 1 .

Tabela 1: Itens após validação de face e conteúdo

\begin{tabular}{|c|c|}
\hline CÓDIGO & DIMENSÃO AUTOEFICÁCIA \\
\hline AEF 1 & Participo da prestação de serviços públicos nos quais eu possua capacidade para realizá-los \\
\hline AEF2 & Participo porque os serviços prestados pelo Estado precisam de melhorias \\
\hline AEF3 & Tenho competência para tomar decisões nas atividades que desenvolvo \\
\hline AEF4 & Minha atuação melhora os serviços públicos ofertados à sociedade \\
\hline CÓDIGO & DIMENSÃO NATUREZA DO SERVIÇO \\
\hline NATS1 & Busco organizações que prestem serviços mais fáceis de realizar \\
\hline NATS2 & Ter informaçôes sobre o serviço que prestarei é fundamental para minha participação \\
\hline NATSB & Conhecer os dirigentes da organizaçăo é fundamental para minha participação no serviço \\
\hline NATS 4 & Procuro me informar sobre o tempo de execução dos serviços para saber se poderei executá-los \\
\hline NATS5 & Participo na prestação de servicos se for realizado perto da comunidade onde moro \\
\hline NATS6 & Participo na prestação de serviços se exigir pouco esforço físico \\
\hline NATS7 & Participo na prestação de serviços se exigir pouco esforço mental \\
\hline NATS8 & Participo na prestação de serviços se eu tiver bastante tempo livre \\
\hline CÓDIGO & DIMENSÃO SOCIABILIDADE \\
\hline SOC1 & Participo na prestação de serviços para não me sentir excluído da sociedade \\
\hline SOC2 & Prefiro participar de serviços que sejam realizados em grupo \\
\hline $\mathrm{SOCB}$ & $\begin{array}{l}\text { A participação de outras pessoas na prestação do serviço, é fundamental para que eu também } \\
\text { participe }\end{array}$ \\
\hline SOC4 & Fazer novas amizades é importante para minha participação \\
\hline CÓDIGO & DIMENSÃO RECOMPENSAS \\
\hline REC1 & Participo da prestação de serviços para aprender algo novo \\
\hline $\mathrm{REC2}$ & Presto esse tipo de serviço para preencher meu tempo vago \\
\hline $\mathrm{REC} 3$ & Minha felicidade aumenta à medida que participo das atividades da organização \\
\hline REC4 & Analiso o que a organização pode me oferecer de benefício antes de me envolver \\
\hline REC5 & Elogios aumentam a minha disposição para a prestação de serviços \\
\hline REC6 & Receber algum brinde ao final da prestação do serviço \\
\hline CÓDIGO & DIMENSÃO RELEVÂNCIA PERCEBIDA \\
\hline RELP1 & Participo dos serviços que tragam melhorias para a comunidade onde moro \\
\hline RELP2 & Engajo-me em serviços que possam melhorar o bem-estar da minha familia \\
\hline RELP3 & Participo na prestaçấo de servicos se for importante para mim \\
\hline RELP4 & Participo na prestação de serviços se for importante para um familiar \\
\hline RELP5 & Participo na prestação de serviços se for importante para um amigo \\
\hline RELP6 & Participo na prestação de serviços se a organização tiver regulamento formal \\
\hline
\end{tabular}

\section{Passo 3 - Decisões sobre as respostas}

Esse passo é caracterizado pela análise das alternativas e a decisão sobre qual escala de averiguação utilizar. A pesquisa utilizou a escala do tipo Likert de 10 pontos. Optou-se pela escala de 10 pontos por favorecer o entendimento do respondente, uma vez que apresenta uma clara indicação de intensidade de concordância entre os dois extremos. Baseando-se em Costa (2011) e Rossiter (2002), foram estabelecidos os seguintes critérios de exclusão para os itens: Correlação menor que 0,3 e maior que 0,9; Medida KMO menor que 0,7; Comunalidade menor que 0,4; Carga fatorial menor que 0,4; Variância de explicação do fenômeno menor que $50 \%$ e Alpha de Cronbach menor que 0,7 .

Passo 4 - Construção do instrumento de pesquisa

$\mathrm{O}$ conjunto de itens foi colocado em um instrumento adequado aos propósitos da pesquisa, com a finalidade de viabilizar a coleta de dados que servirão para as duas etapas seguintes (trabalho de amostragem e limpeza da escala). O questionário foi construído com os 28 itens constantes da Tabela 1.

Foram feitas modificaçóes nos enunciados de alguns itens para melhor entendimento do respondente e melhor mensuração do construto. $\mathrm{O}$ instrumento de pesquisa foi composto pelo título 'motivação para 
coprodução', seguido de uma breve apresentação e a definição de coprodução, com o objetivo de deixar claro para o respondente a temática da pesquisa. O questionário solicitou dados sociodemográficos com a finalidade de conhecer os respondentes e disponibilizou os itens das cinco dimensões de forma aleatória, para não criar um "padrão" de resposta por parte dos respondentes.

\section{Passo 5 - Primeira atividade de amostragem}

A amostra principal para esta pesquisa foi definida como sendo os cidadãos que coproduzem por meio das OSC. Por se tratar do desenvolvimento e validação de uma escala, optou-se por não delimitar geograficamente a amostra. Nesse passo, que se caracteriza com uma atividade com finalidade exploratória, não foram utilizados os sujeitos principais da amostra, deixando-os para o passo 7. Assim, a escala proposta terá passado por duas amostras com o objetivo de obter evidências de validade e confiabilidade consistentes.

O instrumento de coleta de dados para a realização da primeira amostragem foi aplicado com voluntários, em geral, integrantes de diversas OSC, caracterizando, portanto, uma amostra não probabilística. Entendese que para a primeira amostragem, por ser uma fase exploratória, o voluntário poderia ser o respondente, uma vez que o cidadão que coproduz com as OSC poderá estar inserido na amostra.

Para a gestão do trabalho de campo da amostragem optou-se pela coleta mediada, que é a realizada sem a presença física de qualquer agente de coleta. A coleta de dados da primeira amostragem foi realizada no período entre 01 e 20 de outubro de 2019. Durante o período de coleta de dados foram obtidas 370 respostas.

Passo 6 - Procedimentos de limpeza da escala

Realização de procedimentos exploratórios para analisar os resultados da primeira amostragem e proceder à primeira rodada de limpeza da escala. Ou seja, eliminar da escala aqueles itens que não se ajustam bem à dimensão proposta, ou realizar possíveis melhorias no item para um novo teste de amostragem. Inicialmente, detectou-se apenas uma resposta que apresentou missing values, a mesma foi excluída e os dados foram analisados por meio do software estatístico SPSS25. (Statistical Package for Social Sciences). Ainda com relação a avaliação de entradas, foi analisado se algum item recebeu a mesma frequência de resposta e verificou-se que todos os itens tiveram resposta mínima 1 e máxima 10, o que demonstra que nenhum respondente marcou uma resposta única para os itens da escala.

A análise fatorial exploratória (AFE) foi executada mediante a extração de componentes principais, sem a utilização de fixação de números de fatores e com método de rotação Varimax, com base em autovalores superiores a 1 e suprimindo fatores com valor absoluto menor que 0,3 . Ainda foram extraídas as variâncias de explicação do fenômeno, a carga fatorial e as comunalidades. Para análise de confiabilidade foi extraído o alpha de Cronbach e a variação, caso o item seja excluído. A seguir são apresentados os resultados das dimensões.

Os itens da dimensão autoeficácia apresentaram correlação entre 0,3 e 0,9 e $\mathrm{KMO}(0,777)$, sinalizando boa correlação e boa adequação dos itens da dimensão. Todos os itens apresentaram comunalidade e carga fatorial maior que 0,4 , e a variância de explicação do fenômeno foi superior a $60 \%$. A extração do alpha apresenta 0,779 e não sofre alteração com a exclusão de algum item, conforme Tabela 2.

Tabela 2: Dimensão autoeficácia

\begin{tabular}{lccc}
\hline & Comunalidade & Carga fatorial & $\begin{array}{c}\text { Alpha se item } \\
\text { excluído }\end{array}$ \\
\hline AEF1 & 0,619 & 0,787 & 0,723 \\
$\mathrm{AEF} 2$ & 0,600 & 0,775 & 0,728 \\
$\mathrm{AEF3}$ & 0,556 & 0,746 & 0,743 \\
$\mathrm{AEF} 4$ & 0,650 & 0,806 & 0,706 \\
\hline Variância \% & & & 60,616 \\
\hline Alpha de Cronbach & & 0,779 \\
\hline
\end{tabular}


A dimensão natureza do serviço apresentou correlações diversas com NATS1, NATS2, NATS3 e NATS4, abaixo de 0,3. Esses itens apontam para uma possível exclusão na AFE. Após as correlações e o teste KMO $(0,695)$ foi realizada a AFE na dimensão. Nesse procedimento, a matriz de componente rotativa apresentou dois fatores, indicando que a dimensão precisa de ajustes. A variância de explicação do fenômeno foi superior a $50 \%$, porém, carregando mais de um fator. A extração do alpha apresenta 0,720 e não sofre alteração com a exclusão de itens. Os itens que apresentaram correlaçóes baixas foram excluídos para uma nova rodada da AFE.

A exclusão dos itens provocou um aumento do $\operatorname{KMO}(0,750)$ e do alpha $(0,734)$, indicando a adequação dos itens da dimensão. Todos os itens da dimensão apresentam índices dentro do estabelecido para a pesquisa, conforme Tabela 3 .

Tabela 3: Dimensão natureza do serviço

\begin{tabular}{llll}
\hline \multicolumn{2}{l}{ Comunalidade } & $\begin{array}{l}\text { Carga } \\
\text { fatorial }\end{array}$ & $\begin{array}{l}\text { Alpha se item } \\
\text { excluído }\end{array}$ \\
\hline NATS5 & 0,595 & 0,771 & 0,657 \\
NATS6 & 0,634 & 0,796 & 0,640 \\
NATS7 & 0,546 & 0,739 & 0,681 \\
NATS8 & 0,461 & 0,679 & 0,715 \\
\hline KMO & & 0,750 \\
\hline Variância \% & & 55,884 \\
\hline
\end{tabular}

A primeira análise na dimensão sociabilidade apresentou correlações baixas para SOC1 e SOC4, indicando que os itens podem ser excluídos. Em seguida, foi realizado o teste de adequação da amostra $\mathrm{KMO}(0,662)$, sendo necessário ajustar os itens da dimensão para que o valor do teste fique dentro do estabelecido na pesquisa.

O primeiro procedimento de AFE aponta a matriz de componente rotativa com apenas um fator, indicando que os itens da dimensão estão medindo o mesmo construto. O item SOC1 apresentou comunalidade abaixo de 0,4, confirmando, assim, sua exclusão. A extração do alpha apresenta 0,663 e a exclusão do SOC1 provoca seu aumento, corroborando com a decisão de exclusão do item.

Após a exclusão de SOC1 foi realizada a segunda rodada da AFE, que apresentou aumento da variância de explicação do fenômeno e elevação do alpha. Porém, a dimensão apresenta índices de KMO e alpha abaixo dos estabelecidos para a pesquisa. Mesmo com os índices baixos, não foi realizada uma terceira rodada para que a dimensão não apresentasse apenas dois itens. Os dados da segunda rodada estão demonstrados na Tabela 4.

Tabela 4: Dimensão sociabilidade

\begin{tabular}{lllc}
\hline & Comunalidade & Carga fatorial & Alpha se item excluído \\
\hline SOC2 & 0,695 & 0,834 & 0,519 \\
SOC3 & 0,699 & 0,836 & 0,521 \\
SOC4 & 0,470 & 0,685 & $\mathbf{0 , 7 2 4}$ \\
\hline KMO & & $\mathbf{0 , 6 3 0}$ \\
\hline Variância \% & & 62,130 \\
\hline Alpha de Cronbach & & $\mathbf{0 , 6 9 2}$ \\
\hline
\end{tabular}

Por se tratar da primeira versão do questionário e ele não ter respondentes específicos, os itens SOC1 e SOC4 foram reescritos (passo 7) e mantidos na pesquisa por ocasião da realização da segunda amostragem. 
A análise da dimensão recompensas, corrobora com a literatura sobre motivação para a coprodução ao apontar para a baixa incidência das recompensas como propulsoras da coprodução (Petukiene, Tijunaitiene, \& Damkuviene, 2012; Fledderus \& Honingh, 2016; Thijssen \& Van Dooren, 2016). A análise da dimensão inicia apontando uma matriz de correlação com todos os itens da dimensão apresentando, ao menos, uma correlação abaixo de 0,3 , indicando que podem ser excluídos. $\mathrm{O}$ teste de adequação da amostra $\mathrm{KMO}(0,689)$ aponta para a necessidade de ajuste dos itens da dimensão.

Após a análise de correlação e do teste KMO, procedeu-se a AFE que aponta uma matriz de componente rotativa com dois fatores, indicando que os itens da dimensão não estão medindo o mesmo construto e que a dimensão precisa de ajustes. Os itens REC2 e REC 5 carregaram em dois fatores, com cargas fatoriais bem próximas, indicando uma possível exclusão.

O procedimento de análise da variância de explicação do fenômeno ficou muito próximo do limite de exclusão $(57,1 \%)$ e esse percentual leva em consideração a soma dos dois fatores, que não interessa nesta pesquisa. A extração do alpha aponta 0,679 e não se altera com a exclusão de algum item.

Considerando que todos os itens da dimensão apresentaram correlações abaixo de 0,3 ; que o teste $\mathrm{KMO}$ teve resultado abaixo de 0,7; que os itens tiveram cargas fatoriais diversas e em mais de uma dimensão; que o alpha apresenta resultado abaixo de 0,7 e não se altera com a exclusão de nenhum item, optou-se pela exclusão de todos os itens da dimensão recompensas para a elaboração do segundo instrumento de pesquisa.

A análise dos itens da dimensão relevância percebida, começa pela de correlação bivariada de Pearson e aponta índices baixos para os itens RELP1, RELP6 e RELP2. A extração do KMO (0,712) indica que a dimensão pode ser ajustada. A AFE trouxe a matriz de componente rotativa em dois fatores, indicando, mais uma vez, que a dimensão precisa de ajustes. A variância de explicação do fenômeno foi de $67,9 \%$, porém, representa a soma de dois fatores. A extração do alpha apresenta 0,768 e sofre alteração com a exclusão de RELP1 e RELP6.

Para a realização de uma segunda rodada de AFE e a extração do alpha, optou-se pela exclusão dos itens RELP1, RELP2 e RELP6. Após a exclusão, a matriz de componente rotativa apresentou apenas um fator. Os itens apresentam comunalidade e carga fatorial dentro dos limites da pesquisa. A variância de explicação do fenômeno e o alpha de Cronbach apresentaram cargas maiores. Mesmo com a exclusão de RELP3 indicando a elevação do alpha, optou-se pela não exclusão do item, uma vez que o alpha apresenta valor dentro do estabelecido para a pesquisa, conforme a Tabela 5.

Tabela 5: Dimensão relevância percebida

\begin{tabular}{llll}
\hline \multicolumn{2}{l}{ Comunalidade } & $\begin{array}{l}\text { Carga } \\
\text { fatorial }\end{array}$ & $\begin{array}{l}\text { Alpha se item } \\
\text { excluído }\end{array}$ \\
\hline RELP3 & 0,669 & 0,818 & 0,935 \\
RELP4 & 0,894 & 0,946 & 0,744 \\
RELP5 & 0,857 & 0,926 & 0,786 \\
\hline \multicolumn{2}{l}{ Variância \% } & & 80,684 \\
\hline
\end{tabular}

Os itens dessa dimensão, que serão utilizados na segunda coleta de dados, corroboram com estudos que apontam que o cidadão estará mais motivado a participar, quanto mais relevante, para si ou para o próximo, ele perceber o serviço (Pestoff, 2012; Van Eijk \& Steen, 2016; Vanleene et al., 2017).

Para conclusão do passo 6 foi realizada a AFE e extração do alpha de Cronbach com os itens selecionados em cada dimensão. Após a primeira rodada da AFE e extração do alpha com todos os itens, o item SOC4 apresentou carga fatorial similar em duas dimensões e foi excluído. Optou-se por inserir NATS2 na análise com todos os itens, por entender que o item pode apresentar carga fatorial satisfatória na dimensão autoeficácia. 
A Tabela 6 apresenta os resultados da AFE e alpha com todos os itens selecionados após os primeiros procedimentos de limpeza da escala.

Tabela 6: Itens utilizados na segunda coleta

\begin{tabular}{|c|c|c|c|}
\hline \multicolumn{2}{|c|}{ Comunalidade } & \multirow{2}{*}{$\begin{array}{l}\begin{array}{l}\text { Carga } \\
\text { fatorial }\end{array} \\
0,767\end{array}$} & \multirow{2}{*}{$\begin{array}{l}\text { Alpha se item } \\
\text { excluído }\end{array}$} \\
\hline AEF1 & 0,598 & & \\
\hline AEF2 & 0,581 & 0,727 & 0,812 \\
\hline AEF3 & 0,605 & 0,767 & 0,820 \\
\hline $\mathrm{AEF} 4$ & 0,631 & 0,772 & 0,812 \\
\hline NATS2 & 0,517 & $0,642^{*}$ & 0,816 \\
\hline NATS5 & 0,581 & 0,656 & 0,808 \\
\hline NATS6 & 0,696 & 0,822 & 0,815 \\
\hline NATS7 & 0,591 & 0,742 & 0,817 \\
\hline NATS8 & 0,478 & 0,582 & 0,818 \\
\hline $\mathrm{SOC} 2$ & 0,805 & 0,861 & 0,815 \\
\hline SOCB & 0,765 & 0,819 & 0,812 \\
\hline RELP3 & 0,653 & 0,743 & 0,804 \\
\hline RELP4 & 0,880 & 0,901 & 0,800 \\
\hline RELP5 & 0,843 & 0,879 & 0,799 \\
\hline \multicolumn{3}{|c|}{ Número de observações } & 369 \\
\hline \multicolumn{3}{|l|}{$\mathrm{KMO}$} & 0,804 \\
\hline \multicolumn{3}{|c|}{ Variância \% } & 65,884 \\
\hline \multicolumn{3}{|c|}{ Alpha de Cronbach } & 0,824 \\
\hline
\end{tabular}

${ }^{*}$ Carga fatorial em outra dimensão

Concluídos os passos de 1 a 6 na construção da escala, tem-se um conjunto menor e, supostamente, mais consistente e adequado para mensuração da motivação para CBP. Costa (2011) aponta que o primeiro procedimento de limpeza da escala não é suficiente para afirmar que os itens finais são válidos e medem o construto. Assim, é necessário realizar novas atividades de campo com a finalidade de levantar novos dados para aprimorar a escala.

Passo 7 - Trabalhos de campo adicionais

Para a realização desse passo, Costa (2011) sugere a construção de novos questionários, o planejamento de amostragens adicionais e a gestão das atividades de campo adicionais. Durante a elaboração do segundo instrumento de pesquisa, foram analisados os itens excluídos com a finalidade de identificar quais são apontados na literatura como potenciais motivadores para a CBP. Assim, optou-se pela rescrita dos itens SOC1, SOC4, NATS1 e RELP1. O segundo instrumento de coleta de dados foi elaborado com os itens reescritos e os constantes na Tabela 6.

Nesse passo foi delimitada a amostra principal da pesquisa: cidadãos que coproduzem bens públicos mediante OSC. Estudos em coprodução sugerem que as pesquisas sobre motivação sejam realizadas em diferentes tipos de organização. Assim, optamos pelas OSC da área de habitação, saúde, cultura e recreação, educação e pesquisa e assistência social por serem as mais representativas, de acordo com o Mapa das Organizações Civis. Optamos, ainda, pelas áreas de desenvolvimento e defesa de direitos, meio ambiente e reciclagem por serem áreas abordadas no contexto brasileiro (Salm, Menegasso, \& Ribeiro, 2007; Ribeiro, Andion, \& Burigo, 2015; Silva, Knol, \& Moretto, 2016; Ronconi, Debetir, \& Mattia, 2011).

A coleta de dados da segunda amostragem foi realizada no período entre 12 de novembro e 14 de dezembro de 2019. Durante o período de coleta de dados foram obtidas 174 respostas. Em relação ao tamanho adequado da amostra, pode-se considerar a quantidade de itens e respondentes sugeridos por Hair, Black, Babin, Anderson e Tatham, (2009), de que o tamanho da amostra deve conter cinco vezes o número de itens. A pesquisa apresenta uma relação superior a nove vezes o número de respondentes por itens.

Passo 8 - Procedimentos adicionais de limpeza da escala 
Nesse passo os procedimentos foram aplicados sobre os dados da segunda amostra com análises exploratórias preliminares, análise de correlação das variáveis e a análise fatorial confirmatória, conforme apresentadas a seguir.

\section{Análise Exploratória Preliminar}

Após a segunda coleta de dados, foi realizada a análise por meio do Missing Value Analysis, que atestou a validade das 174 respostas. Ainda com relação a avaliação de entradas, foi analisado se algum item recebeu a mesma frequência de resposta e verificou-se que todos os itens tiveram resposta mínima 1 e máxima 10, o que demonstra que nenhum respondente marcou uma resposta única para todos os itens.

\section{Dados sociodemográficos da amostra}

A amostra foi formada por 174 respondentes, sendo $61,5 \%$ de mulheres e $38,5 \%$ de homens. A maioria dos respondentes estão na faixa etária entre 30 e 40 anos, perfazendo um total de 37,4\%. Referente ao grau de escolaridade dos respondentes prevaleceu um grau alto, com 41,4\% possuindo nível superior incompleto/ completo. A renda familiar mensal dos respondentes ficou entre $\mathrm{R} \$ 1.000,00$ e $\mathrm{R} \$ 3.000,00$, com um percentual de $41,4 \%$.

Com o objetivo de melhor caracterizar a amostra, foi perguntado a área de atuação da organização na qual o cidadão coproduzia. As áreas com maior número de participantes foram a saúde e assistência social, respectivamente com $46,6 \%$ e $19,5 \%$. Destacamos que o gênero masculino apresenta maioria nas áreas desenvolvimento e defesa de direitos $(72,7 \%)$ e assistência social $(58,8 \%)$. Ressaltamos, também, que a maioria dos coprodutores da área de educação e pesquisa apresentam menos de 30 anos, enquanto que na área de saúde, a maioria possui entre 30 e 40 anos. Podemos dizer, de maneira geral, que a amostra apresenta características heterogêneas adequadas para as análises desenvolvidas nesse passo.

Análise de correlação, consistência interna e análise fatorial exploratória e análise fatorial confirmatória.

Com o objetivo de realizar os procedimentos adicionais de limpeza da escala, foi feita a análise de correlação bivariada de Person, a análise de consistência interna e a análise fatorial exploratória e confirmatória dos itens da escala. A realização da extração das correlações aponta a menor delas $(0,058)$ entre NATS2 e NATS6. O alpha de Cronbach extraído foi de 0,921 , que indica bom ajuste dos itens para medir a motivação para coprodução. A variância de explicação do fenômeno é de $68,1 \%$.

As cargas fatoriais foram extraídas utilizando o método da máxima verossimilhança (Maximum Likelihood) com os itens apresentando cargas fatoriais acima de 0,4 em pelo menos uma dimensão. No entanto, os itens SOC3 e SOC4 apresentaram cargas fatoriais muito similares em mais de uma dimensão. O mesmo acontecendo com RELP1 e RELP3. Optou-se por não excluir itens após a segunda AFE, uma vez que as dimensões sociabilidade e relevância percebida ficariam com dois itens e inviabilizaria a realização da AFC.

Para a realização da AFC foram definidos os índices de ajustamento. Os valores de referência variam na literatura, foram adotados índices baseados em Marôco (2010) e Hair et al. (2009). Foram estabelecidos os seguintes critérios de aceitação: GFI (Índice de qualidade de ajuste) maior que 0,900; CFI (Índice de ajuste comparativo) maior que 0,900; TLI (Índice de Tucker-Lewis) maior que 0,900; RMSEA (Raiz do erro quadrático médio de aproximação) menor que $0,08, \chi^{2} / \mathrm{gl}$ (qui-quadrado sobre grau de liberdade) menor que 5; carga fatorial maior que 0.700 e variância explicada acima de 0.500 .

A AFC iniciou pela dimensão autoeficácia, que teve acrescida na análise o item NATS2. Para o modelo ajustado, optou-se pela exclusão de AEF2 por apresentar carga fatorial menor que 0,7 . A dimensão apresenta medidas finais dentro dos padrões estabelecidos, conforme Tabela 7. 
Tabela 7: Análise Confirmatória - Dimensão autoeficácia

\begin{tabular}{|c|c|c|c|c|c|}
\hline \multicolumn{3}{|l|}{ CÓDIGO } & \multicolumn{3}{|c|}{ CARGA FATORIAL } \\
\hline \multicolumn{3}{|c|}{ AEF1 AEF2 AEF3 AEF4 NATS2* } & \multicolumn{3}{|l|}{$\begin{array}{l}0,732 \\
0,592 \\
0,829 \\
0,771 \\
0,737\end{array}$} \\
\hline \multicolumn{6}{|c|}{ MEDIDAS FINAIS DA DIMENSÃO } \\
\hline GFI 0.968 & CFI 0.968 & TLI 0.904 & & RMSEA 0.070 & $\begin{array}{l}2 / g l \\
0.003\end{array}$ \\
\hline
\end{tabular}

* O item foi alterado para AEF5

$\mathrm{Na}$ realização da AFC da dimensão natureza do serviço, todos os itens apresentaram carga fatorial satisfatória e medidas finais dentro dos limites estabelecidos, indicando boa adequação dos itens, segundo a Tabela 8.

Tabela 8: Análise Confirmatória - Dimensão natureza do serviço

\begin{tabular}{|c|c|c|c|c|c|}
\hline \multicolumn{3}{|l|}{ CÓDIGO } & \multicolumn{3}{|c|}{ CARGA FATORIAL } \\
\hline \multicolumn{3}{|c|}{$\begin{array}{l}\text { NATS } 1 \text { NATS5 NATS } 6 \text { NATS7 } \\
\text { NATS8 }\end{array}$} & \multicolumn{3}{|c|}{$\begin{array}{l}0,760 \\
0,746 \\
0,929 \\
0,924 \\
0,816\end{array}$} \\
\hline \multicolumn{6}{|c|}{ MEDIDAS FINAIS DA DIMENSÃO } \\
\hline GFI 0.974 & $\begin{array}{l}\text { CFI } \\
0.989\end{array}$ & TLI & & RMSEA 0.004 & $x_{2} / g l 0.033$ \\
\hline
\end{tabular}

A dimensão sociabilidade apresentou na AFE índices abaixo dos estabelecidos nos itens SOC1 e SOC4 e foi preciso reescrevê-los para a segunda amostragem. Por ocasião da AFC, todos os itens apresentaram cargas fatoriais satisfatórias e a dimensão parece ajustada ao modelo, de acordo com a Tabela 9.

Tabela 9: Análise Confirmatória - Dimensão sociabilidade

\begin{tabular}{|c|c|c|c|c|c|}
\hline \multicolumn{3}{|l|}{ CÓDIGO } & \multicolumn{3}{|c|}{ CARGA FATORIAL } \\
\hline \multicolumn{3}{|c|}{ SOC1 SOC2 SOC3 SOC4 } & \multicolumn{3}{|l|}{$\begin{array}{l}0,705 \\
0,759 \\
0,739 \\
0,809\end{array}$} \\
\hline \multicolumn{6}{|c|}{ MEDIDAS FINAIS DA DIMENSÃO } \\
\hline GFI 1.000 & CFI 1.000 & TLI 1.023 & & $\begin{array}{l}\text { RMSEA } \\
0.009\end{array}$ & $x 2 / g l 0.999$ \\
\hline
\end{tabular}

A última dimensão analisada na AFC é a relevância percebida. O item RELP1 foi excluído por apresentar carga fatorial de 0,642. Os demais itens apresentam carga fatorial satisfatória. A Tabela 10 apresenta os resultados da dimensão. 
Tabela 10: Análise Confirmatória - Dimensão relevância percebida

\begin{tabular}{|c|c|c|c|c|c|}
\hline \multicolumn{3}{|l|}{ CÓDIGO } & \multicolumn{3}{|c|}{ CARGA FATORIAL } \\
\hline \multicolumn{3}{|c|}{ RELP1 RELP3 RELP4 RELP5 } & \multicolumn{3}{|l|}{$\begin{array}{l}0,642 \\
0,703 \\
0,971 \\
0,937\end{array}$} \\
\hline \multicolumn{6}{|c|}{ MEDIDAS FINAIS DA DIMENSÃO } \\
\hline GFI 0.997 & CFI 1.000 & TLI 1.005 & & RMSEA 0.673 & $\begin{array}{l}x^{2} / g l \\
0.549\end{array}$ \\
\hline
\end{tabular}

A dimensão apresenta níveis aceitáveis de ajustamento, mesmo com o RMSEA $(0,673)$ indicando ajustamento. Hair et al. (2009) apontam que esse coeficiente pode variar em modelos complexos, como é o caso do modelo que se pretende desenvolver nesta pesquisa.

A conclusão do passo 8 é a apresentação dos itens que serão submetidos à validação e confiabilidade. Assim, após a AFC realizada dimensão por dimensão, optou-se pela exclusão dos itens AEF2 e RELP1. Com a exclusão dos itens e do agrupamento de NATS2, foram realizadas as análises de ajustes e discrepâncias.

A análise fatorial confirmatória com os itens agrupados apresenta uma série de ajustes a serem realizados, seja por carga fatorial, variância explicada ou índices de ajustes para criação da escala. O GFI (0.746), CFI (0.820), TLI (0.786) e RMSEA (0.135) apresentam índices fora dos estabelecidos para a pesquisa, indicando que os itens analisados não formam uma escala ajustada para mensuração da motivação para a CBP.

Para ajuste dos índices foram excluídos os itens AEF1, AEF5, SOC2 e RELP3 por apresentarem cargas fatoriais abaixo de 0.700 e nível de explicação do fenômeno abaixo de 0.500 . A Tabela 11 apresenta o conjunto de itens que serão submetidos à validade e confiabilidade.

Tabela 11: Validade e confiabilidade

\begin{tabular}{|c|c|c|c|}
\hline CÓDIGO & CARGA FATORIAL & & VARIÂNCIA EXPLICADA \\
\hline $\begin{array}{l}\text { AEF3 AEF4 NATS1 NATS5 } \\
\text { NATS6 NATS7 NATS8 SOC1 } \\
\text { SOC3 SOC4 RELP4 RELP5 }\end{array}$ & $\begin{array}{l}0.850 \\
0.799 \\
0.769 \\
0.761 \\
0.918 \\
0.917 \\
0.828 \\
0.720 \\
0.766 \\
0.767 \\
0.949 \\
0.959\end{array}$ & & $\begin{array}{l}0.723 \\
0.638 \\
0.591 \\
0.579 \\
0.843 \\
0.841 \\
0.685 \\
0.519 \\
0.587 \\
0.588 \\
0.900 \\
0.920\end{array}$ \\
\hline \multicolumn{4}{|l|}{ MEDIDAS FINAIS DOS ITENS } \\
\hline CFI 0.928 & TLI 0.905 & RMSEA 0.077 & $\times 2 / g l 0.000$ \\
\hline
\end{tabular}

Os itens que serão submetidos à análise de validade e de confiabilidade para a criação da escala, apresentam índices dentro do estabelecido para a pesquisa.

Passo 9 - Análise de validade e de confiabilidade da escala final

Para Costa (2011), esse passo pode ser considerado o mais relevante na construção de uma escala, uma vez que será dada a confirmação que o esforço de construção gerou uma escala com características de validade e confiabilidade. Foi verificada a validade convergente e discriminante e a confiabilidade da escala mediante extração do alpha de Cronbach e o Ômega.

A validade convergente aponta se os itens de uma escala possuem variância média extraída satisfatória dentro da dimensão (Hair et al., 2009; Costa, 2011). Para Hair et al. (2009), uma validade convergente adequada deve apresentar variância média extraída superior a 0,5 . O exame da confiabilidade no conjunto 
final de itens, tem por finalidade verificar se a escala pode ser considerada confiável em termos de consistência interna. Para verificação da consistência interna das dimensões foi extraído o alpha de Cronbach e ômega de McDonald.

Tabela 12: Validade convergente e confiabilidade

\begin{tabular}{l|l|l|l|l}
\hline & AEF & NATS & SOC & RELP \\
\hline Avevar & 0.678 & 0.658 & 0.569 & 0.679 \\
\hline Alpha & 0.810 & 0.889 & 0.792 & 0.952 \\
\hline Ômega & 0.807 & 0.910 & 0.796 & 0.945 \\
\hline
\end{tabular}

Pela análise de confiabilidade e validade convergente, verifica-se, na Tabela 12, que em todas as dimensões o valor de ômega e alpha são maiores que 0,7 e o avevar, ou a variância média extraída, é maior que 0,5.

A validade discriminante serve para analisar o quanto um construto é diferente dos demais (Hair et al., 2009). A análise da validade discriminante compara as variâncias extraídas para cada dimensão - valores em negrito, na diagonal da Tabela 13 - e as variâncias compartilhadas. As variâncias extraídas são obtidas por meio da raiz quadrada dos valores de avevar e precisam ser maiores que os valores para as variâncias compartilhadas entre a dimensão analisada e as demais. A Tabela 13 aponta as variâncias extraídas em cada dimensão.

Tabela 13: Validade discriminante

\begin{tabular}{l|l|l|l|l}
\hline & AEF & NATS & SOC & RELP \\
\hline AEF & $\mathbf{0 , 8 2 3}$ & & & \\
\hline NATS & 0,233 & $\mathbf{0 , 8 1 1}$ & & \\
\hline SOC & 0,568 & 0,628 & $\mathbf{0 , 7 5 4}$ & \\
\hline RELP & 0,444 & 0,593 & 0,523 & $\mathbf{0 , 9 5 3}$ \\
\hline
\end{tabular}

Após a realização dos procedimentos de validação e confiabilidade, a escala para motivação da CBP é apresentada na tabela 14 .

Tabela 14: Escala proposta

\begin{tabular}{|c|c|}
\hline DIMENSÃO & ITENS \\
\hline \multirow{2}{*}{ AUTOEFICÁCIA } & Tenho competência para tomar decisões nas atividades que desenvolvo \\
\hline & Minha atuaçẫo melhora os serviços públicos ofertados à sociedade \\
\hline \multirow{5}{*}{ NATUREZA DO SERVIÇO } & Procuro organizações que prestem serviços fáceis de realizar \\
\hline & Participo na prestação de serviços se for realizado perto da comunidade onde moro \\
\hline & Participo na prestação de serviços se exigir pouco esforço físico \\
\hline & Participo na prestaçấo de serviços se exigir pouco esforço mental \\
\hline & Participo na prestação de serviços se eu tiver bastante tempo livre \\
\hline \multirow[b]{2}{*}{ SOCIABILIDADE } & Participo para me sentir membro ativo na sociedade \\
\hline & $\begin{array}{l}\text { A participação de outras pessoas na prestação do serviço, é fundamental para que eu também participe } \\
\text { Participo para fazer novas amizades }\end{array}$ \\
\hline RELEVÂNCIA PERCEBIDA & Participo na prestação de serviços se for importante para um familiar \\
\hline
\end{tabular}

A escala inicial era composta de 28 itens e cinco dimensões. No entanto, a escala final tem 12 itens e quatro dimensões. Os resultados encontrados nesta pesquisa servem para corroborar com pesquisas realizadas em 
outros países, mostrando que os cidadãos engajados na CBP não o fazem com o intuito de receber elogios, não estão buscando felicidade ou prestando serviços em troca de benefícios materiais. Esses aspectos restam evidenciados nos itens da escala.

O instrumento proposto apresenta itens relacionados com as recompensas intrínsecas (Alford, 2002), aspectos não materiais (Pestoff, 2012; Van Eijk \& Steen, 2014), a natureza do serviço (Petukiene et al., 2012), bem como itens relacionados a competência e ao "fazer a diferença" quando o cidadão se envolve na CBP (Vanleene et al., 2015; Uzochukwu \& Thomas, 2018). As dimensões propostas corroboram com a literatura existente e trazem elementos não apresentados em outros estudos, como o aspecto de uma pessoa ser motivada a coproduzir porque outra pessoa coproduz e a participação na CBP buscando fazer novas amizades. Esses aspectos podem ser explorados pelos gestores no desenho de políticas de participação

\section{Passo 10 - Desenvolvimento de normas e recomendações}

A escala de motivação para CBP deve ser utilizada para mensurar os aspectos que motivam o cidadão brasileiro a coproduzir mediante organizações da sociedade civil. Porém, poderá ser utilizada para mensurar a motivação de cidadãos nos diversos contextos da coprodução. A escala poderá, ainda, sofrer adaptações e ser aplicada em diversos cenários nos quais ocorram a participação cidadã.

Para aplicação da escala, Costa (2011) recomenda que o conjunto de itens seja disposto no questionário de forma aleatória evitando associações entre as variáveis, de preferência não mais que 10 itens por seção, para evitar descontinuidade nas respostas ou enviesar a pesquisa.

\section{CONSIDERAÇÕES FINAIS}

Esta pesquisa se propôs a contribuir empírica e teoricamente com o campo da motivação para a coprodução do bem público, apresentando uma resposta para a seguinte pergunta de pesquisa: $\mathrm{O}$ que motiva o cidadão a participar na CBP por meio das OSC? Para que houvesse uma resposta satisfatória, foi delimitado como objetivo principal a construção de uma escala para mensuração dos aspectos que motivam o cidadão a participar da CBP. No processo de construção da escala foram abordados diversos aspectos e, ao propor e validar a mesma, apresentamos o que parece ser os principais aspectos que motivam o engajamento do cidadão nas ações coprodutivas do Estado, por meio das OSC. Como existem diferentes perspectivas sobre o engajamento do cidadão na coprodução, as OSC e o Estado necessitam de um instrumento capaz de identificar os aspectos motivacionais que possibilite selecionar os cidadãos e fazer com que permaneçam engajados.

A escala pode facilitar a implementação do processo de coprodução, por refletir aspectos motivacionais do cidadão e apontar que não há um modelo ou formato único para os diferentes contextos da coprodução. Os resultados encontrados neste estudo mostram que diferentes contextos provocam motivações diversas no cidadão que coproduz. Existe, por exemplo, uma percepção de esforço do cidadão que é relevante para seu engajamento na CBP, quando o mesmo aponta que busca organizações que prestem serviços fáceis de realizar; que sua motivação para coproduzir é maior se a prestação do serviço for realizada perto de sua residência e se a prestação de serviços exigir pouco esforço físico e/ou mental.

Os resultados mostram, também, que o cidadão será motivado a coproduzir se entender que o serviço prestado será relevante para a sociedade em geral, ou especificamente, para um familiar, um amigo. Assim, ao recrutar cidadãos para a $\mathrm{CBP}$, os gestores das OSC devem enfatizar a relevância do serviço a ser prestado. Outro apontamento é sobre a motivação do cidadão em coproduzir para se sentir membro ativo da sociedade, para fazer novas amizades e sua motivação baseada na participação de outras pessoas. Esses aspectos demonstram que gestores devem investir na divulgação dos serviços prestados por sua OSC. Isso fará com que o cidadão que participa da OSC sinta que está prestando um bom serviço à sociedade e trará outros cidadãos para coproduzirem naquela OSC. Entendemos que os gestores podem adotar diferentes estratégias destinadas ao envolvimento dos cidadãos a partir dos resultados encontrados na pesquisa. 
Não podemos deixar de apresentar uma limitação da pesquisa aplicada por meio eletrônico. Ficou claro, em algumas situações, que os respondentes não tinham habilidade com celular ou computador. Acreditase que alguns cidadãos deixaram de responder ao questionário devido à forma de coleta de dados, fazendo diminuir a amostra da pesquisa.

Por fim, como sugestão de novos estudos, é possível a realização de uma pesquisa para testar a validade da escala em uma OSC de área específica. Sugere-se, também, que sejam desenvolvidos estudos sobre motivações individuais para CBP, uma vez que itens com tais características apresentaram scores maiores. Apesar de alguns estudos apontarem motivações intrínsecas e extrínsecas para o cidadão coproduzir, analisálas conjuntamente fez com os itens fossem excluídos da composição da escala. Assim, sugere-se que estudos futuros possam analisar aspectos intrínsecos e extrínsecos separadamente.

Para concluir, espera-se que esta pesquisa sirva de inspiração e fundamentação para outros estudos com o objetivo de ampliar o conhecimento sobre a motivação para coprodução do bem público no Brasil e fortalecer esse campo de pesquisa.

\section{REFERÊNCIAS}

Age, L. M., Schommer, P. C. (2017). Coprodução de Serviço de Vigilância Sanitária: Certificação e Classificação de Restaurantes. Revista de Administração Contemporânea, 21(3), 413-434. https://doi.org/10.1590/1982-7849r ac2017170026.

Alford, J. (2002). Why Do Public-Sector Clients Coproduce?: Toward a Contingency Theory. Administration \& Society, 34(1), 32-56. doi.org/10.1177/0095399702034001004

Alford, J., Yates, S. (2016). Co - Production of Public Services in Australia: The Roles of Government Organizations and Co - Producers. Australian Journal of Public Administration, 75, 159-175. Doi: 10.1111/1467-8500.12157

Bovaird, T., Ryzin, V. G., Loeffler, E., Parrado, S. (2015). Activating Citizens to Participate in Collective CoProduction of Public Services. Journal of Social Policy, 44(1), 1-23. DOI: 10.1017/S0047279414000567

Brandsen, T., Pestoff, V. (2006) Co-production, the third sector and the delivery of public services, Public Management Review, 8(4), 493-501. https://doi.org/10.1080/14719030601022874

Cepiku, D., Giordano, F. (2014). Co-Production in Developing Countries: Insights from the community health workers experience, Public Management Review, 16(3), 317-340. https://doi.org/10.1080/14719037.2013.82 2535

Chaebo, G., Medeiros, J. (2017). Participação direta em coprodução é democrática? Controle vetorial da dengue em Campo Grande - MS. Cadernos Gestão Pública e Cidadania, 22(71), 1-20. DOI: 10.12660/cgpc.v22n71.61067

Costa, F. J. (2011). Mensuração e Desenvolvimento de Escalas. Rio de Janeiro: Editora Ciência Moderna.

Fledderus, J., Brandsen, T., Honingh, M. E. (2015). User co-production of public service delivery: An uncertainty approach. Public Policy and Administration, 30(2), 145-164. https://doi.org/10.1177/0952076715572362

Fledderus, J., Honingh, M. (2016). Why people co-produce within activation services: the necessity of motivation and trust - an investigation of selection biases in a municipal activation programme in the Netherlands. International Review of Administrative Sciences, 82(1), 69-87. https://doi.org/10.1177/0020852314566006

Hair, J. F., Black, W. C., Babin, B. J., Anderson, R. E., Tatham, R. L. (2009). Análise multivariada de dados. (6a ed.). Porto Alegre: Bookman Editora.

Klein, V. H. Jr., Salm, J. F., Heidemann, F. G., Menegasso, M. E. (2012). Participação e coprodução em política habitacional: estudo de um programa de construção de moradias em SC. Revista de Administração Pública, 46(1), 25-48. http://dx.doi.org/10.1590/S0034-76122012000100003.

Marôco, J (2010). Análise de equaçôes estruturais: Fundamentos teóricos, software \& aplicações. Report number, Pêro Pinheiro.

Ostrom, E. (1996). Crossing the great divide: Coproduction, synergy, and development. World Development, 24(6), 1073-1087. https://doi.org/10.1016/0305-750X(96)00023-X 
Pestoff, V. (2012). Co-production and third sector social services in Europe - Some crucial conceptual issues. In V. Pestoff, T. Brandsen, \& B. Verschuere (Eds.), New public governance, the third sector and co-production (Chap. 2). London: Routledge.

Petukiene, E., Tijunaitine, R., Damkuviene, M. (2012). Participation of clients in public services: the aspect of motivating. Inzinerine Ekonomika-Engineering Economics, 23(3), 301-309. DOI:10.5755/j01.ss.73.3.788

Ribeiro, A., Andion, C., Burigo, F. (2015). Ação coletiva e coprodução para o desenvolvimento rural: um estudo de caso do Colegiado de Desenvolvimento Territorial da Serra Catarinense. Revista de Administração Pública, 49(1), 119-140. DOI: 10.1590/0034-76121685

Rocha, A., Schommer, P. C., Debetir, E., Pinheiro, D. (2019). Transparência como elemento da coprodução na pavimentação de vias públicas. Cadernos Gestão Pública e Cidadania, 24(78), 1-22. DOI: https://doi.org/10.1 2660/cgpc.v24n78.74929

Ronconi, L. F. A., Debetir, E., Mattia, C. (2011). Conselhos Gestores de Políticas Públicas: potenciais espaços para a coprodução dos serviços públicos. Contabilidade, Gestão e Governança, 14(3), 46-59.

Rossiter, J. R. (2002). The C-OAR-SE procedure for scale development in marketing. International Journal of Research in Marketing, 19(4), 305-335. https://doi.org/10.1016/S0167-8116(02)00097-6

Salm, J. F., Menegasso, M. E., Ribeiro, R. M. (2007). Co-produção do bem público e o desenvolvimento da cidadania: o caso do Proerd em Santa Catarina. Revista Alcance, 14(2), 231-246. DOI: https://doi.org/10.14210/alcance .v14n2.p231-246

Schommer, P. C., Nunes, J. T., Moraes, R. L. (2012). Accountability, controle social e coprodução do bem público: a atuação de vinte observatórios sociais brasileiros voltados à cidadania e à educação fiscal. Publicaçôes da Escola da AGU: Gestão Pública Democrática, Brasília, (4)18, 229-258.

Silva, F. A., Knoll, A., Moretto, L. Neto. (2016). A Coprodução de Serviço Público na Reciclagem de Resíduos Sólidos: Estudo no Município de Florianópolis. Revista de Administração, Contabilidade e Economia da FUNDACE, 7(3), 64-76. DOI: http://dx.doi.org/10.13059/racef.v7i3.388

Soares, G., Farias, J. (2019). Com quem a escola pode contar? A coprodução do Ensino Fundamental público por familiares de estudantes. Revista de Administração Pública, 53(2), 310-330. http://dx.doi.org/10.1590/0034-7 61220170301

Thijssen, P., Van Dooren, W. (2016). Who you are/where you live: do neighborhood characteristics explain coproduction? International Review of Administrative Sciences, 82(1), 88-109. https://doi.org/10.1177/0020852 315570554

Uzochukwu, K., Thomas, J.C. (2018), Who Engages in the Coproduction of Local Public Services and Why? The Case of Atlanta, Georgia. Public Admin Rev, 78, 514-526. https://doi.org/10.1111/puar.12893

Van Eijk, C. J. A., Steen, T. P. S. (2014). Why People Co-Produce: Analyzing citizens' perceptions on co-planning engagement in health care services, Public Management Review, 16(3), 358-382. https://doi.org/10.1080/147 19037.2013.841458

Van Eijk, C. J. A., Steen, T. P. S. (2016). Why engage in co-production of public services? Mixing theory and empirical evidence. International Review of Administrative Sciences, 82(1), 28-46. https://doi.org/10.1177/0020852314 566007

Vanleene, D., Verschuere, B., Voets, J. (2017). Co-Producing a Nicer Neighborhood: Why Do People Participate in Community Development Projects? Paper presented at the IIAS Study Group on Co-Production, Nijmegen. DOI: https://doi.org/10.4335/15.1.111-132(2017)

Verschuere, B., Brandsen, T., Pestoff, V. (2012). "Co-Production: The State of the Art in Research and the Future Agenda.” Voluntas 23 (4): 1083-1101. DOI: 10.1007/s11266-012-9307-8 